\title{
Clinical Study \\ Case Series of Monoamniotic and Pseudomonoamniotic Twin Gestations
}

\author{
Shunji Suzuki \\ Department of Obstetrics and Gynecology, Japanese Red Cross Katsushika Maternity Hospital, 5-11-12 Tateishi, \\ Katsushika-ku, Tokyo 124-0012, Japan \\ Correspondence should be addressed to Shunji Suzuki; czg83542@mopera.ne.jp
}

Received 20 December 2012; Accepted 23 January 2013

Academic Editors: E. Ginsburg and H. Rehder

Copyright (c) 2013 Shunji Suzuki. This is an open access article distributed under the Creative Commons Attribution License, which permits unrestricted use, distribution, and reproduction in any medium, provided the original work is properly cited.

\begin{abstract}
We retrospectively evaluated a series of 18 monoamniotic and 7 pseudomonoamniotic (secondary to rupture in the membrane dividing monochorionic diamniotic twins) twin gestations managed after 20 weeks' gestation. There were no significant differences in the incidence of neonatal death or umbilical cord entanglement between the monoamniotic and pseudomonoamniotic twin gestations (33 versus $21 \%, P=0.94$ and 72 versus $43 \%, P=0.36$ ). Therefore, the same serious management may be needed for pseudomonoamniotic twin gestations as for monoamniotic twin gestations.
\end{abstract}

\section{Introduction}

Monoamniotic twinning is a rare complication, occurring in less than $1 \%$ of monozygosity and is associated with a significant mortality rate $[1,2]$. The most common cause of perinatal mortality in monoamniotic twins has been reported to be cord entanglement $[1,2]$. Cord entanglement has been reported in up to $70 \%$ of monoamniotic twins with $50 \%$ or more of deaths attributed to this complication [3]. Recently, some cases of disruption of the dividing membrane in monochorionic diamniotic twin gestation as pseudomonoamniotic twin gestation [4-13]. In some previous reports, pseudomonoamniotic twin gestation sometimes has been also observed to be complicated by umbilical cord entanglement. In addition, some cases of monochorionic diamniotic twin pregnancy complicated by spontaneous antepartum rupture of the intertwin-dividing membrane (and umbilical cord entanglement) without any perinatal episodes have been reported $[12,13]$; however, there have been few investigations comparing the perinatal outcomes in pseudomonoamniotic twins with those in true monoamniotic twins. Therefore, in this study, we examined the perinatal outcomes of these pregnancies.

\section{Methods}

We retrospectively evaluated a series of 18 monoamniotic and 7 pseudomonoamniotic twin gestations managed after 20 weeks' gestation at our hospital (these contain the cases we reported previously $[6-8,14])$. The diagnosis of monoamnionicity and pseudomonoamnionicity was determined on the basis of ultrasound features and it was confirmed by clinical presentations at delivery, such as the presence of disruption of the dividing membrane and histological examination of the placenta. The histology of the placenta and umbilical cord entanglement were examined at the time of delivery. Cases of congenital anomalies such as anencephaly and acardiac twins were excluded. In addition, the pregnancies were excluded, if fetal demise at least one twin at $<20$ weeks' gestation was diagnosed. The gestational age of the pregnancies was established by ultrasonographic examination of the fetal crown-rump length at 8-11 weeks' gestation.

In our hospitals, elective cesarean at preterm for (true and pseudo-) monoamniotic twin gestations as previously reported $[15,16]$ was not performed without maternal request, if fetuses were well and the patient had no maternal complications. In monoamniotic twin gestations, twin-twin 
TABLE 1: Summary of perinatal findings in 18 monoamniotic twin gestations.

\begin{tabular}{|c|c|c|c|c|c|}
\hline Number & GA at delivery & Twin A & Twin B & UC entanglement & Other complications \\
\hline 1 & 20 & IUFD & IUFD & Yes & \\
\hline 2 & 22 & IUFD & IUFD & Yes & \\
\hline 3 & 28 & $\mathrm{CP}$ & IUFD & Yes & \\
\hline 4 & 28 & Neonatal death & IUFD (22 weeks) & No & Oligohydramnios following polyhydramnios \\
\hline 5 & 29 & $\mathrm{CP}$ & Healthy & Yes & Hydrops \\
\hline 6 & 30 & IUFD (26 weeks) & Healthy & Yes & \\
\hline 7 & 31 & Healthy & Healthy & Yes & \\
\hline 8 & 32 & Healthy & Healthy & No & \\
\hline 9 & 32 & IUFD (28 weeks) & PVL & No & Hydrops (IUFD), polyhydramnios \\
\hline 10 & 33 & IUFD & IUFD & Yes & \\
\hline 11 & 34 & Healthy & Healthy & Yes & \\
\hline 12 & 35 & Healthy & Healthy & Yes & \\
\hline 13 & 36 & Healthy & Healthy & Yes & \\
\hline 14 & 36 & Healthy & Healthy & Yes & \\
\hline 15 & 37 & Healthy & Healthy & Yes & \\
\hline 16 & 37 & Healthy & Healthy & No & \\
\hline 17 & 38 & Healthy & Healthy & Yes & \\
\hline 18 & 39 & Healthy & IUFD (20 weeks) & No & \\
\hline
\end{tabular}

GA: gestational age; UC: umbilical cord; IUFD: intrauterine fetal death; CP: cerebral palsy; PVL: periventricular leukomalacia.

TABLE 2: Summary of perinatal findings in 7 pseudomonoamniotic twin gestations.

\begin{tabular}{lccccc}
\hline Number & GA at delivery & Twin A & Twin B & UC entanglement & Cause of membrane rupture \\
\hline 1 & 27 & IUFD & IUFD & No & Amnioreduction due to TTTS \\
2 & 28 & Healthy & IUFD (21 weeks) & Yes & Single IUFD \\
3 & 31 & Healthy & Healthy & No & FLP due to TTTS \\
4 & 33 & Healthy & Healthy & No & FLP due to TTTS \\
5 & 35 & Healthy & Healthy & Yes & Unknown \\
6 & 36 & Healthy & Healthy & Yes & Unknown \\
7 & 37 & Healthy & Healthy & Unknown \\
\hline
\end{tabular}

GA: gestational age; UC: umbilical cord; IUFD: intrauterine fetal death; TTTS: twin-twin transfusion syndrome; FLP: fetoscopic laser photocoagulation.

transfusion syndrome (TTTS) was diagnosed with the clinical presentations, such as cardiac dysfunction, discordance in bladder size, and/or polyhydramnios.

Cases and controls were compared by the $\chi^{2}$ or Fisher's exact test for categorical variables. Differences with $P<0.05$ were considered significant.

\section{Results}

Table 1 shows the summary of perinatal findings in the 18 (true) monoamniotic twin gestations. The incidence of perinatal loss per total number of neonates was 33\% (12/36). Four cases (22\%) resulted in double death, while $4(22 \%)$ cases resulted in single death. The incidence of cerebral injury per total number of lived neonates was $13 \%(3 / 24)$. The incidence of umbilical cord entanglement was $72 \%(13 / 18)$. There were no cases of severe congenital heart anomalies. Based on the clinical presentations, 2 cases (11\%: cases 4 and 9) were suggested to be complicated by TTTS.
Table 2 shows the summary of perinatal findings in the 7 pseudomonoamniotic twin gestations. In 3 cases of these $(43 \%)$, the disruption of the dividing membrane was associated with the therapies (amniocentesis and fetoscopic laser photocoagulation) for TTTS. As our impression, at the deliveries the area of the perforated portions of dividing membranes seemed to be large enough to lead to umbilical cord entanglement. Of 4 cases with spontaneous membrane rupture, 2 mothers (cases 5 and 7) felt the amniotic fluid flow due to membrane rupture of the second twin before labor. The incidence of perinatal loss per total number of neonates was $21 \%(3 / 14)$. One case $(14 \%)$ resulted in double death, while $1(14 \%)$ case resulted in single death during the second trimester. The incidence of umbilical cord entanglement was $43 \%(3 / 7)$. There were no cases of cerebral injury or severe congenital heart anomalies.

There were no significant differences in the incidence of neonatal death or umbilical cord entanglement between the monoamniotic and pseudomonoamniotic twin gestations 
$(P=0.94$ and 0.36$)$. Thirteen of 15 perinatal losses $(87 \%)$ were occurred before 32 weeks of gestation.

\section{Discussion}

To date, some possible mechanisms leading to antepartum rupture of the intertwin-dividing membrane except artificial septostomy, such as amniocentesis and fetoscopic laser photocoagulation, have been proposed, such as infection (chorioamnionitis) [9], developmental disturbance [9], trauma or physical rupture by fetuses [10], and intrauterine sling formation [11]. In addition, some cases of spontaneous antepartum rupture, in which the exact cause of rupture of membrane cannot be well determined, have been reported. In our 7 cases of pseudomonoamniotic twin gestations, 3 cases were artificial and 4 cases were spontaneous.

We know that the sample size of this study is very small; however, the current results may indicate that the perinatal outcomes of pseudomonoamniotic twin gestations do not differ from those of true monoamniotic twin gestations. Based on the results, the incidence of umbilical cord entanglement, which is the most critical concern in twin gestations, in monoamniotic twins seemed to be similar to that in pseudomonoamniotic twins. As our impression, the area of the perforated portions of dividing membranes seemed to be large enough to lead to umbilical cord entanglement. In addition, there was no significant difference in perinatal loss between the monoamniotic and pseudomonoamniotic twins. Therefore, the same serious management may be needed for pseudomonoamniotic twin gestations as for monoamniotic twin gestations.

In this study, the total incidence of perinatal death in monoamniotic and pseudomonoamniotic twin gestations seemed to be high as previously reported $[1,2]$; however, $87 \%$ of perinatal losses were occurred before 32 weeks of gestation, and there was no occurrence of new perinatal deaths after 34 weeks of gestation. In some literatures, the recommended timing of delivery range to prevent sudden intrauterine fetal death has been reported to be between 32 and 34 weeks $[1,15,16]$. However, the current result may support the other articles that the incidence of fetal death after 32 weeks is not high in monoamniotic twins $[17,18]$, which is suggesting that prophylactic preterm delivery may not be indicated in all monoamniotic twin gestations. Therefore, a further large study may be needed concerning the appropriate timing of delivery for monoamniotic and pseudomonoamniotic twins.

\section{Conclusion}

The same management is needed for monoamniotic and pseudomonoamniotic twin gestations.

\section{References}

[1] K. E. Hack, J. B. Derks, A. H. Schaap et al., "Perinatal outcome of monoamniotic twin pregnancies," Obstetrics and Gynecology, vol. 113, no. 2, pp. 353-360, 2009.

[2] J. E. Dickinson, "Monoamniotic twin pregnancy: a review of contemporary practice," Australian and New Zealand Journal of Obstetrics and Gynaecology, vol. 45, no. 6, pp. 474-478, 2005.
[3] L. L. Su, "Monoamniotic twins: diagnosis and management," Acta Obstetricia et Gynecologica Scandinavica, vol. 81, no. 11, pp. 995-1000, 2002.

[4] G. A. Aisenbrey, V. A. Catanzarite, T. J. Hurley, J. H. Spiegel, D. B. Schrimmer, and A. Mendoza, "Monoamniotic and pseudomonoamniotic twins: sonographic diagnosis, detection of cord entanglement, and obstetric management," Obstetrics and Gynecology, vol. 86, no. 2, pp. 218-222, 1995.

[5] K. J. Lee, M. K. Kim, S. Y. Lee, W. S. Lee, and Y. H. Lee, "Spontaneous rupture of thedividing membrane in a monochorionic pregnancy resulting in a pseudo-monoamniotic pregnancy with cord entanglement," Journal of Obstetrics and Gynaecology Research, vol. 38, pp. 863-866, 2012.

[6] N. Miura and S. Suzuki, "Fetal asphyxia due to cord entanglement in a monochorionic diamniotic twin pregnancy complicated by 2 nd-trimester single intrauterine demise," Fetal Diagnosis and Therapy, vol. 23, no. 1, pp. 69-71, 2008.

[7] S. Suzuki, G. Ishikawa, R. Sawa, Y. Yoneyama, Y. Otsubo, and T. Araki, "Iatrogenic monoamniotic twin gestation with progressive twin-twin transfusion syndrome," Fetal Diagnosis and Therapy, vol. 14, no. 2, pp. 98-101, 1999.

[8] T. Tamura, A. Miura, and S. Suzuki, "Spontaneous disruption of the dividing membrane in monochorionic diamniotic twin pregnancy," Fetal Diagnosis and Therapy, vol. 30, pp. 241-242, 2011.

[9] W. M. Gilbert, S. E. Davis, C. Kaplan, D. Pretorius, T. A. Merritt, and K. Benirschke, "Morbidity associated with prenatal disruption of the dividing membrane in twin gestations," Obstetrics and Gynecology, vol. 78, no. 4, pp. 623-630, 1991.

[10] S. E. Chen, L. Trupin, and S. Trupin, "Antepartum rupture of diamniotic membranes separating monozygotic twins: a case report," Journal of Reproductive Medicine for the Obstetrician and Gynecologist, vol. 39, no. 1, pp. 67-70, 1994.

[11] J. E. De Lia and D. Worthington, "Intrauterine sling with umbilical cord entanglement in diamniotic twins," Ultrasound in Obstetrics and Gynecology, vol. 15, no. 5, p. 447, 2000.

[12] F. K. Nasrallah and Y. A. Faden, "Antepartum rupture of the intertwin-dividing membrane in monochorionic diamniotic twins: a case report and review of the literature," Prenatal Diagnosis, vol. 25, no. 9, pp. 856-860, 2005.

[13] K. Yoshimura, Y. Aiko, H. Inagaki, M. Nakata, and T. Hachisuga, "Prenatal spontaneous disruption of the dividing membrane in monochorionic diamniotic twins detected at the time of fetoscopic laser photocoagulation," Journal of Obstetrics and Gynaecology Research, vol. 35, no. 6, pp. 1129-1131, 2009.

[14] S. Suzuki, K. Kaneko, S. Shin, and T. Araki, "Incidence of intrauterine complications in monoamniotic twin gestation," Archives of Gynecology and Obstetrics, vol. 265, no. 2, pp. 57-59, 2001.

[15] E. Beasley, G. Megerian, A. Gerson, and N. S. Roberts, "Monoamniotic twins: case series and proposal for antenatal management," Obstetrics and Gynecology, vol. 93, no. 1, pp. 130134, 1999.

[16] H. Roqué, J. Gillen-Goldstein, E. Funai, B. K. Young, and C. J. Lockwood, "Perinatal outcomes in monoamniotic gestations," Journal of Maternal-Fetal and Neonatal Medicine, vol. 13, no. 6, pp. 414-421, 2003.

[17] J. A. Tessen and F. J. Zlatnik, "Monoamniotic twins: a retrospective controlled study," Obstetrics and Gynecology, vol. 77, no. 6, pp. 832-834, 1991.

[18] S. R. Carr, M. P. Aronson, and D. R. Coustan, "Survival rates of monoamniotic twins do not decrease after 30 weeks' gestation," American Journal of Obstetrics and Gynecology, vol. 163, no. 3, pp. 719-722, 1990. 


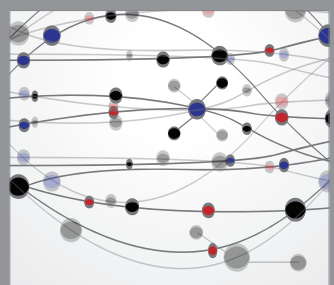

The Scientific World Journal
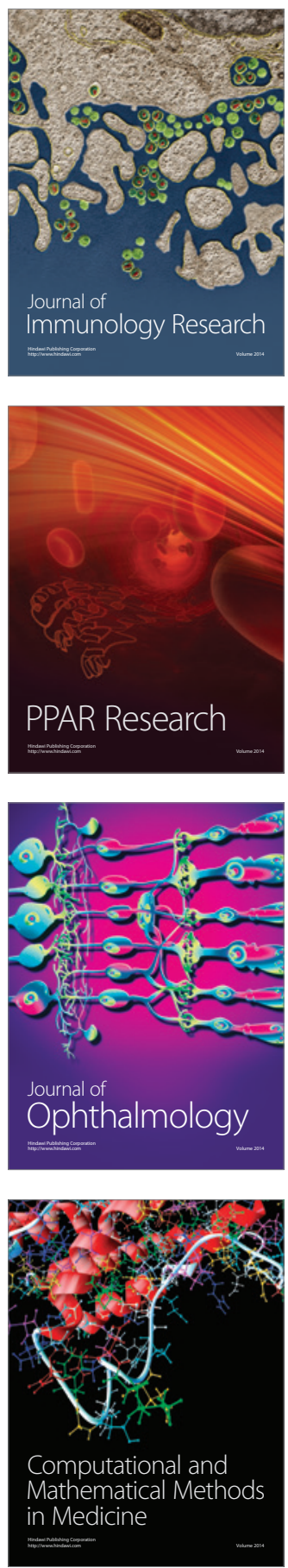

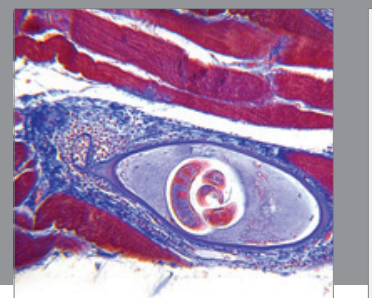

Gastroenterology

Research and Practice
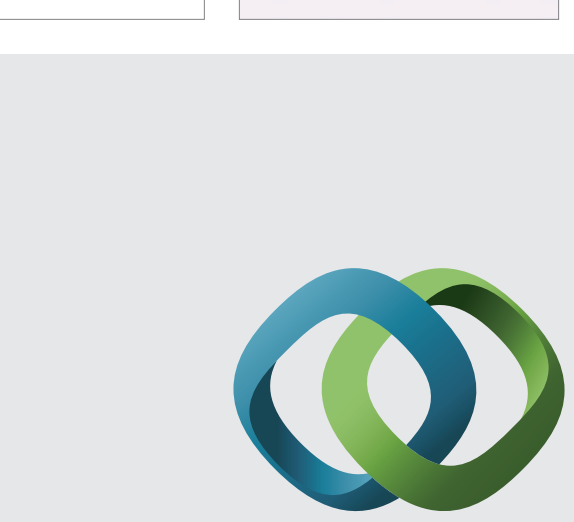

\section{Hindawi}

Submit your manuscripts at

http://www.hindawi.com
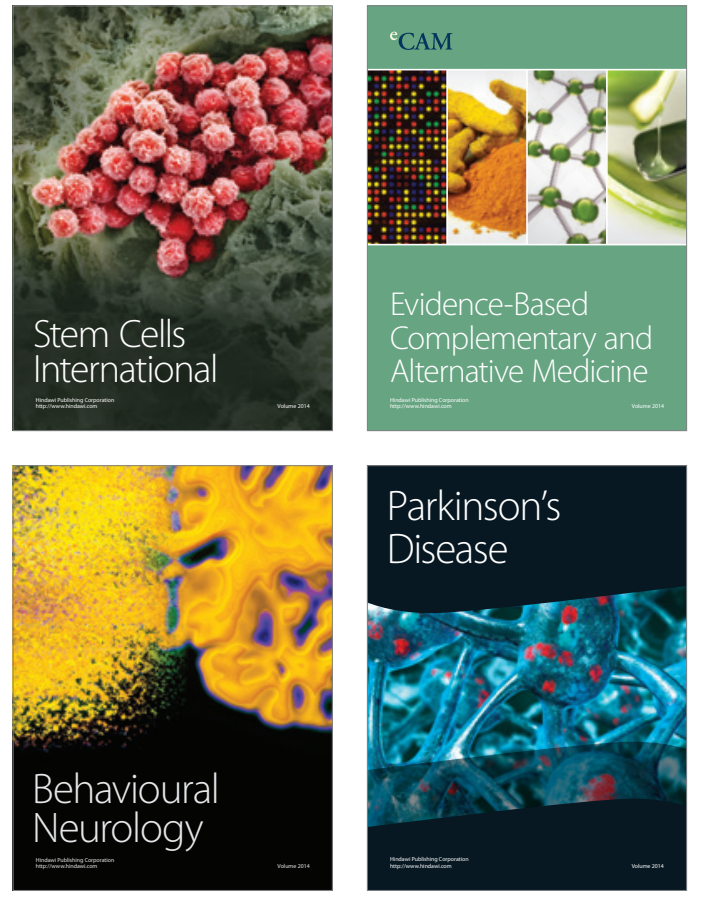
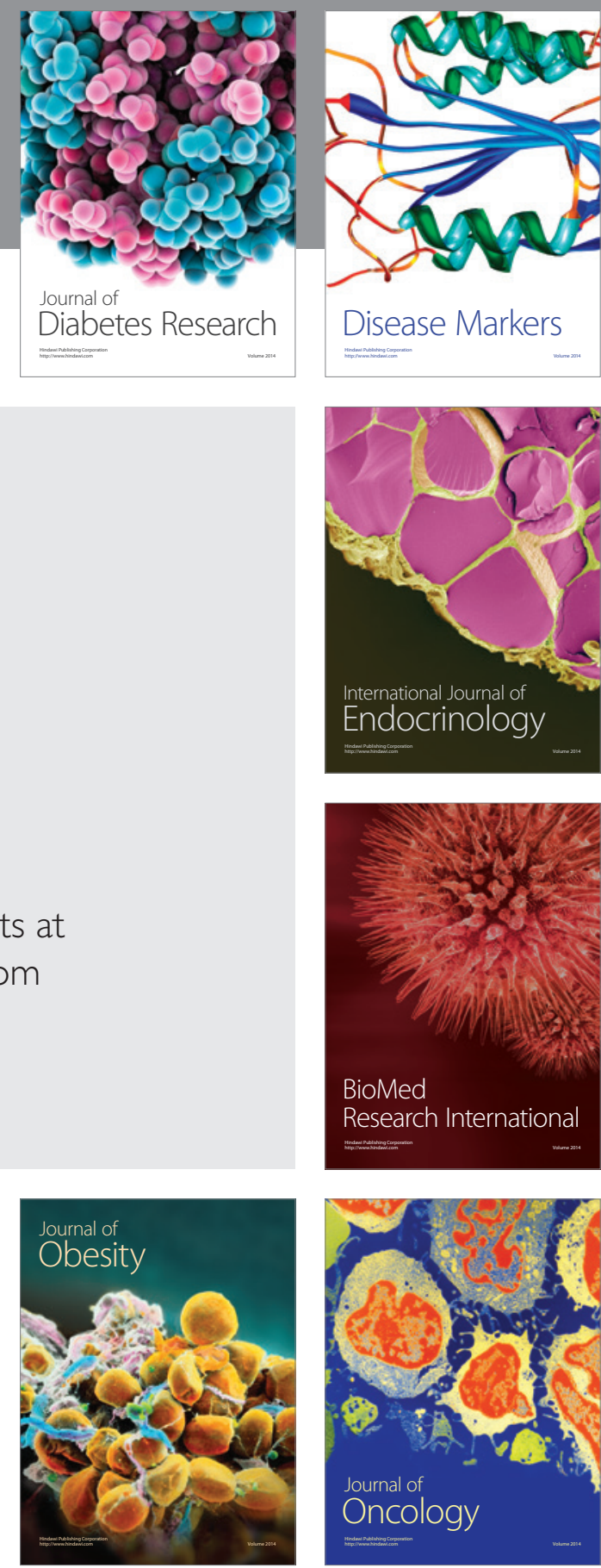

Disease Markers
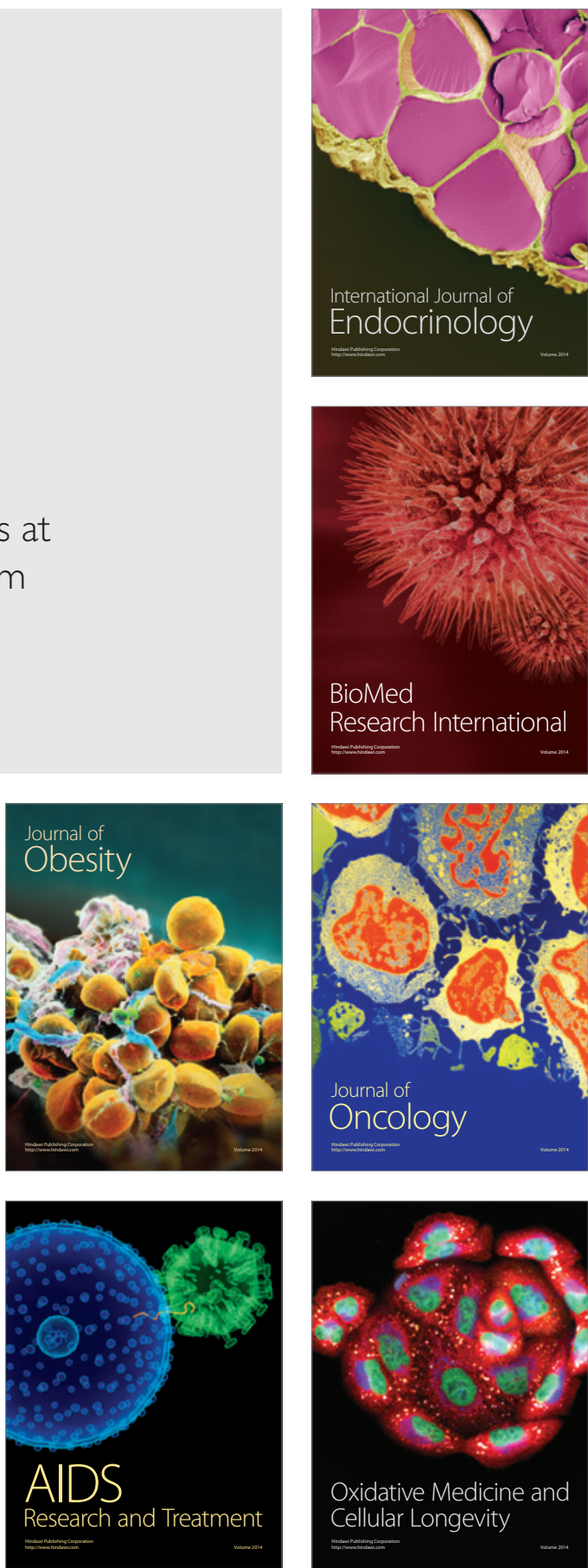